Modelling single positron tracks in $\mathrm{Ar}$

This article has been downloaded from IOPscience. Please scroll down to see the full text article.

2012 J. Phys. B: At. Mol. Opt. Phys. 45045207

(http://iopscience.iop.org/0953-4075/45/4/045207)

View the table of contents for this issue, or go to the journal homepage for more

Download details:

IP Address: 150.203.177.185

The article was downloaded on 14/02/2012 at 02:55

Please note that terms and conditions apply. 


\title{
Modelling single positron tracks in Ar
}

\author{
R P McEachran ${ }^{1}$, J P Sullivan ${ }^{1}$, S J Buckman ${ }^{1}$, M J Brunger ${ }^{2,3}$, \\ M C Fuss ${ }^{4}$, A Muñoz ${ }^{5}$, F Blanco ${ }^{6}$, R D White ${ }^{7}$, Z Lj Petrović ${ }^{8}$, \\ P Limão-Vieira ${ }^{9}$ and G García ${ }^{4}$ \\ ${ }^{1}$ ARC Centre for Antimatter-Matter Studies, Australian National University, Canberra, ACT 0200, \\ Australia \\ ${ }^{2}$ ARC Centre for Antimatter-Matter Studies, School of Chemical and Physical Sciences, \\ Flinders University, GPO Box 2100, Adelaide, South Australia 5001, Australia \\ ${ }^{3}$ Institute of Mathematical Sciences, University of Malaya, Kuala Lumpur 50603, Malaysia \\ ${ }^{4}$ Instituto de Física Fundamental, Consejo Superior de Investigaciones Científicas (CSIC), \\ Serrano 113-bis, 28006 Madrid, Spain \\ ${ }^{5}$ Centro de Investigaciones Energéticas Medioambientales y Tecnológicas (CIEMAT), Avenida \\ Complutense 22, 28040 Madrid, Spain \\ ${ }^{6}$ Departamento de Física Atómica, Molecular y Nuclear, Universidad Complutense de Madrid, \\ 28040 Madrid, Spain \\ ${ }^{7}$ ARC Centre for Antimatter-Matter Studies, James Cook University, Townsville, QLD 4810, \\ Australia \\ ${ }^{8}$ Institute of Physics, University of Belgrade, PO Box 68, 11080 Belgrade, Serbia \\ ${ }^{9}$ Departamento de Física, CEFITEC, FCT-Universidade Nova de Lisboa, 2829-516 Caparica, \\ Portugal \\ E-mail: g.garcia@iff.csic.es
}

Received 12 December 2011, in final form 10 January 2012

Published 7 February 2012

Online at stacks.iop.org/JPhysB/45/045207

\begin{abstract}
In this study, we present a complete set of positron interaction cross sections for scattering from $\mathrm{Ar}$, for incident energies ranging from 0 to $10 \mathrm{keV}$. Experimental data have been critically reviewed from previous experiments performed at the Australian National University and University College London. Differential and integral cross sections, including the effect of positronium formation, have been calculated by using two different optical potential methods. The results of these calculations, in combination with experimental cross sections and experimental energy-loss spectra, have been established as input parameters for an event-by-event Monte Carlo simulation procedure to generate single positron tracks in argon. The reliability of this method to obtain energy deposition models at the nano-scale is also discussed.
\end{abstract}

(Some figures may appear in colour only in the online journal)

\section{Introduction}

Due to the efficiency of PET scanners detecting premature tumours and cancer metastasis, positron-emitting sources are increasingly used in nuclear medicine [1]. The resolution of these systems has been recently improved by new medical imaging techniques and developments. However, positron sources used in medicine are beta emitters which show a wide energy distribution from very low energies up to the $\mathrm{MeV}$ domain with typical average energies of hundreds of $\mathrm{keV}$. Since positron annihilation mainly occurs at relatively low energies, below $100 \mathrm{eV}$, modelling positron tracks from emission energies down to final annihilation, in biologically relevant media, can be useful to try and improve PET resolution and in designing more efficient radiation sources [2]. This type of model represents a real challenge for fundamental research, as it will need both differential and integral interaction cross sections for all the processes taking place across the whole energy range. Unfortunately, the available databases for positron interactions with atoms and molecules typically fail this criterion, so that a systematic study both from the experimental and theoretical points of view is required to improve this situation. Note also, that as incident positrons thermalize through interactions with the atoms and molecules, 
secondary electrons are increasingly likely to be produced. As a consequence, complete cross-section databases for electron impact interactions are also needed if one is to build a complete picture of positron transport and its consequences for radiation damage.

With this paper, we start a systematic study of positron interaction cross sections with atoms and molecules, with the general purpose of obtaining comprehensive, evaluated collisional data sets to model positron tracks in biomolecular systems. We will focus first on Ar, since it is a relatively simple atomic target for which reliable electron experimental and theoretical data are accessible [3, 4]. In addition, very recently some detailed experimental cross-section data have become available from the Australian National University (ANU) [5, 6] by using a 'Surko'-type buffer-gas trap and beam apparatus [7], from University College London (UCL) [8], from the University of Trento [9] as well as the compilation from Sukavov et al [10]. The effects of anisotropic scattering on transport properties have also been recently studied [11]. Once establishing this experimental positron data, we will propose two-model potential calculation procedures to complete the required cross-section information from 0 to $10 \mathrm{keV}$. Above this energy, we will assume that the BornBethe theory can be applied $[12,13]$ to obtain collision cross sections and energy losses in terms of generalized oscillator strength distribution functions.

Finally, single positron tracks in Ar will be simulated with an event-by-event Monte Carlo procedure, which uses our derived collisional data as input parameters.

\section{Data requirements and analysis}

A detailed modelling procedure, such as we are proposing here, requires interaction probability distribution functions, both as a function of energy and scattering angle, as well as energy-loss distribution functions for the overall energy range. In principle, this is the case from 0 up to the maximum energy emitted by the positron radioactive source, typically about $1 \mathrm{MeV}$. The BornBethe theory, as a consequence of the first Born approximation, represents a drastic simplification of the problem. Certainly, when it applies, the interactions of positrons and electrons are identical and the double differential cross sections, as a function of angle and energy loss, can be represented in terms of Bethe surfaces (generalized oscillator strength distribution functions). They then provide integral cross-section values by simple integration. We have shown $[14,15]$ that, in the case of electrons, this approximation overestimates the total cross sections, and especially the elastic part, even at $5 \mathrm{keV}$ for simple atoms and molecules. This is also likely to be true for positrons. We therefore consider it unreasonable to use this approximation below $10 \mathrm{keV}$, and it is in this region where we will focus our data analysis efforts. The main parameters which are required below $10 \mathrm{keV}$ can be summarized as follows.

(i) The total positron scattering cross section (TCS). This represents the sum of all the possible interaction processes, and thus is a critical reference value. It also defines the mean free path for track simulation purposes. Available experimental data will be discussed in section 3, and the calculations described in section 4 will complement these data to cover the whole energy range.

(ii) Elastic cross sections. Experimental data for elastic positron processes are scarce. Differential and integral elastic cross sections for positrons in Ar will be the main objective of the calculations described in section 4 .

(iii) Ionization and positronium formation. Above their respective thresholds they represent the main open inelastic channels. The difficulty of incorporating these channels into the calculations, and the existence of reliable experimental data suggests using the latter for the simulations, in combination with analytical extrapolations.

(iv) Other inelastic processes. By combining the total inelastic cross sections calculated in this study, with those mentioned above for ionizing processes, information about the remaining inelastic channels can be derived through reference to the TCS. In the case of argon, these remaining values will be entirely attributed to discrete excitation processes.

(v) Energy-loss distribution functions. At this stage of the investigation, no specific positron energy-loss distribution function can be derived. Thus, we will assume that the energy transferred by the positrons to the target, through inelastic collisions, follows the same trend as that observed for the electrons. This assumption seems reasonable as the main expected difference, i.e. positronium formation, rapidly leads to positron annihilation without any contribution to the energy-loss spectrum

\section{Experimental data}

The main body of experimental data for this study comes from the Centre for Antimatter-Matter Studies, and has been measured using the positron beam-line facility at the ANU. Systematic measurements of positron scattering from Ar have been performed in the last three years from 0 to $60 \mathrm{eV}$. Elastic, positronium formation and total scattering cross sections for positrons scattering in this energy range have been measured for all the noble gases in order to achieve benchmark data for biomedical and industrial applications. The experimental set-up and measurement procedures have been previously described in detail [16]. The elastic and total scattering cross sections, as well as the positronium formation cross section for Ar have been recently published [5, 6], including a detailed analysis of experimental uncertainties and applied corrections. We note that further evidence in support of this choice, at the TCS level, came from independent measurements made at the University of Trento [9]. They found good agreement with the TCS measurements from the ANU even at the very lowest common energies. Positronium formation is one of the main features of positron interactions in matter and has been extensively studied in the last 20 years [17-23]. In order to account for these measurements, we have introduced into the discussion, a summary of experimental results 


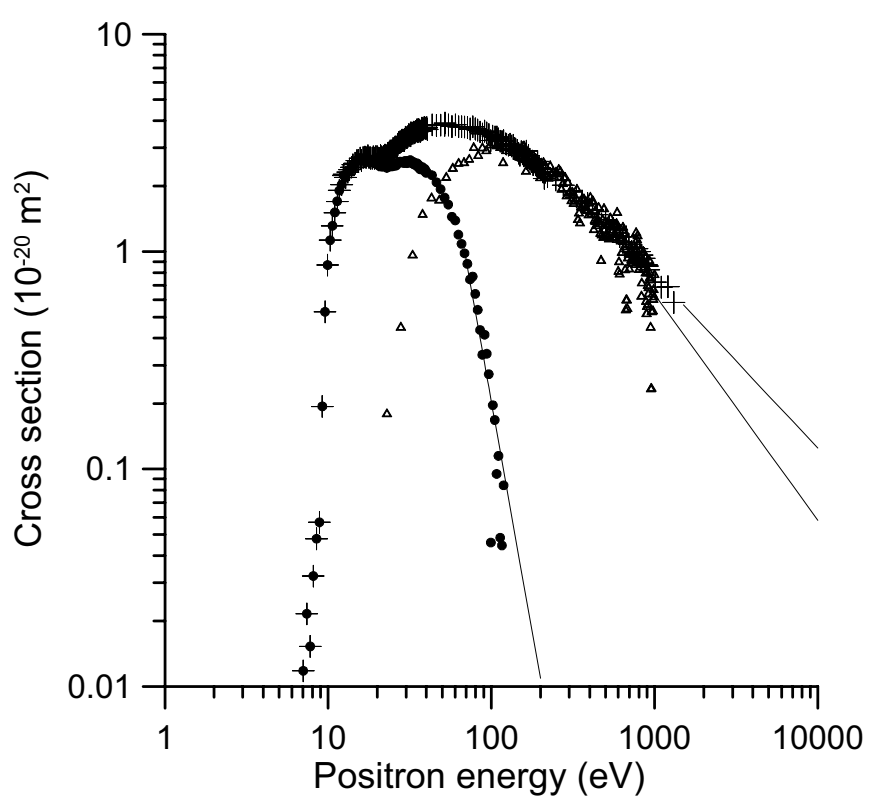

Figure 1. Experimental ionization cross section by positron impact data provided by Laricchia [8]: + , total ionisation; $\Delta$, direct ionisation; $\bullet$, positronium formation. Solid lines represent extrapolated values to higher energies.

from UCL provided by Laricchia [8] as well as their most representative direct ionization cross-section measurements. The experimental data considered in the present study are shown in figures 1 and 2.

The other important process required to track positron interactions is the energy-loss distribution function. By assuming that no energy is transferred to the medium through elastic processes (the mass difference between the positrons and argon atoms makes this quantity negligible), we will need to know the energy-loss distribution function for inelastic collisions. As mentioned above, the most remarkable difference between inelastic collisions involving electrons and positrons is the positronium formation channel. However, once positronium is formed, the positron-electron pair quickly annihilates by losing all its energy and forming $\gamma$-rays. Other inelastic processes, such as direct ionization or electronic excitation, can be considered as binary collisions with the target electrons, and we can assume that the energy-loss distribution function is similar to that derived from electronscattering experiments. Under this assumption, the energyloss distribution function used in this study is an average of those observed for electrons interacting with argon at different energies. The accuracy and limitations of this average energyloss distribution function have been discussed elsewhere [24], so we do not repeat that detail here.

\section{Theoretical calculations}

In order to obtain a complete data set for positron interactions with $\mathrm{Ar}$, some analogies with the case of electron scattering can be assumed. As far as the total scattering cross sections are concerned, figure 2 shows the measured and calculated

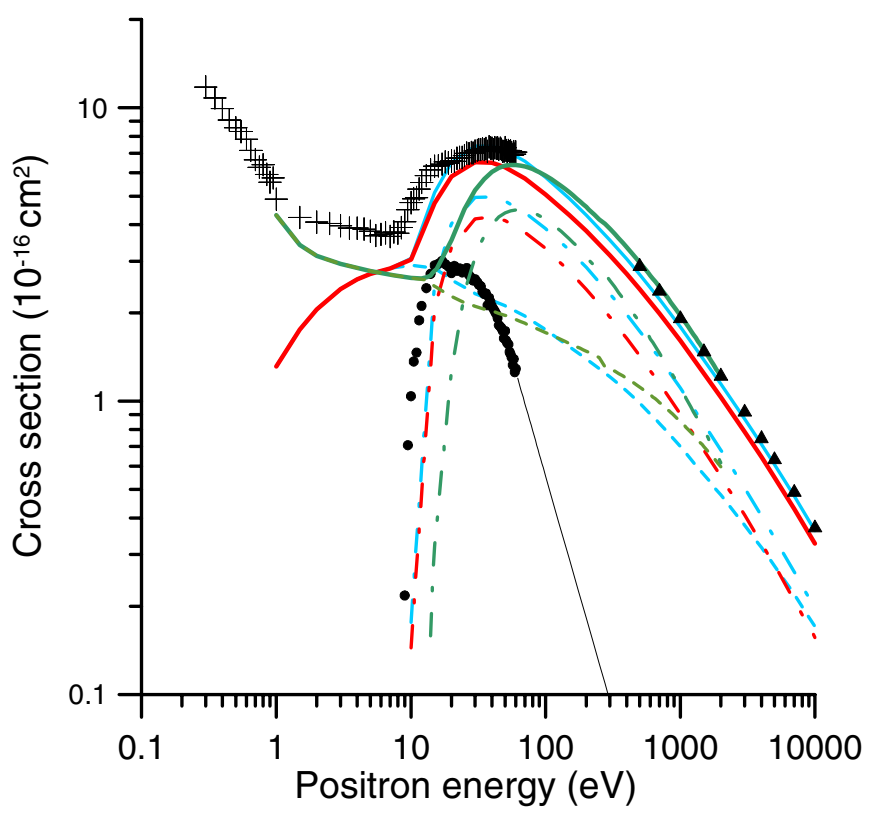

Figure 2. Positron interaction cross sections for argon. Present theoretical and experimental data: + , experimental total scattering cross sections; • , experimental prositronium formation cross sections; - - positronium formation data extrapolated to higher energies; $\boldsymbol{\Delta}$, experimental total electron-scattering cross section from [35]; , calculated total cross sections with the 'ab initio' absorption potential; _-_, integral elastic cross section derived from this potential; $\_\cdot-\bullet_{-}$, integral inelastic cross section derived from this potential; _ calculated total cross sections with the 'quasi-free' absorption potential using as threshold absorption energy the threshold energy for positronium formation; $-_{-{ }_{-}{ }^{\circ}}$, integral inelastic cross section derived from this potential; calculated total cross sections with the 'quasi-free' absorption potential using as threshold energy that for the positronium formation at low energies, below $100 \mathrm{eV}$, and the one corresponding to the first excited state for higher energies; $\ldots--$, integral elastic cross section derived from this potential; ${ }_{-} \cdot \bullet_{-} \cdot$, integral inelastic derived from this potential.

cross-section data for positrons, as well as high-energy $(0.5-5 \mathrm{keV})$ experimental data for electrons, colliding with Ar. Before describing the calculational methods we used, note that, as expected, electron and positron total cross sections tend to merge at high energies. We will consider them equivalent for energies above $10 \mathrm{keV}$, and therefore scattering data for positrons at these energies will be directly taken from electronscattering databases [3]. Below $10 \mathrm{keV}$, particular calculations for positrons are needed and we have used two different methods. Both are based on the formulation of suitable optical potentials, in order to solve the scattering equations for the corresponding complex phase shifts. The main difference between these methods is due to the different procedures used to derive the imaginary (absorption) part of the potential. The first approach is based on the corrected quasi-free absorption potential we have used for electrons [25] by following the method proposed by Reid and Wadehra [26, 27] for positrons. On the other hand, the second approach is an $a b$ initio potential derived by considering the individual excited and ionized states which are accessible at a given incident energy [28]. We can briefly describe the main characteristics of these methods as follows. 


\subsection{Optical potential approach using a quasi-free model for the absorption part}

According to the optical potential model, positron-atom scattering can be represented by the following complex potential [26]:

$$
V_{\text {opt }}(r)=V_{\text {st }}(r)+V_{\text {pol }}(r)+\mathrm{i} V_{\text {abs }}(r),
$$

where $V_{\text {st }}(r)$ is the static potential derived from a standard Hartree-Fock calculation of the atomic charge density, $V_{\mathrm{pol}}(r)$ is the polarization potential and $V_{\mathrm{abs}}(r)$ is the absorption potential that accounts for the inelastic processes. Both are obtained with a similar procedure as explained in [26]. According to this representation, inelastic processes come from binary collisions between incident particles and a 'quasifree' electron cloud representing the target electrons. This scheme has been successfully used for different electronscattering scenarios. We have previously pointed out [25] how critical the definition of the threshold absorption value $(\Delta)$ actually is. In order to be consistent with the representation used for electron collisions, it should be the first excited state of the target. However, around this energy the situation for positrons and electrons is much different. The threshold for positronium formation is given by the ionization limit of the target minus $6.8 \mathrm{eV}$ [17], which normally results in an energy, which is less than that for the first excited state. For this reason, Reid and Wadehra [26, 27] proposed to employ a similar absorption potential to that used for electrons, but moving the absorption threshold $(\Delta)$ down to the positronium formation threshold. This procedure can be regarded as an indirect way to introduce the positronium formation channel into the absorption potential. Results obtained with that approach are shown in figure 2. As illustrated in this figure, these calculations agree very well with experimental data even in the positronium formation region. However, by looking at the experimental positronium formation cross section, an important aspect appears which mitigates against this last procedure. This process actually occurs over a relatively short energy range, from threshold up to about $100 \mathrm{eV}$, while the effect produced by lowering the absorption potential threshold extends over the whole energy range. This means that we are now probably overestimating the total cross section at high energies. An indication of this situation is that convergence with the electron-scattering data is reached at a relatively low energy, about $1 \mathrm{keV}$. In addition, as illustrated by Charlton and Humberston [28], positronium formation at high energy can be considered as a doubly binary collision process. Apart from the binary collision with a target electron, it requires an additional positron or electron scattering off of the residual ion. This indicates that there is part of the process which has been already accounted for by our previous absorption potential (using the excitation threshold). This situation makes it really difficult to introduce the positronium formation as an independent inelastic process. Taking these considerations into account, we propose to adopt here a compromise solution, of using in the calculation the positronium formation threshold up to about $100 \mathrm{eV}$ and the electronic excitation one above this energy. In order to properly overlap both regions, the transition between them occurs gradually, while approaching to $100 \mathrm{eV}$ with the energy dependence derived from that given by the experimental positronium formation cross sections. Results obtained with this last procedure are also plotted in figure 2 . Although differences between these three different choices of the absorption threshold are not very important compared with the accuracy, which we can expect for these calculations ( $\pm 10 \%$ ), we consider the most physical representation to be the third one.

\subsection{Ab initio absorption potential}

The other method we used to derive the absorption potential is completely $a b$ initio and is based upon the excited bound and continuum states of the particular atom as determined in Dirac-Fock calculations [29, 30]. Our potential is thus energy dependent. It becomes non-zero when the incident positron energy equals the first excited-state energy of the atom and more and more terms are added to the potential as the incident energy increases and more excited states and eventually, continuum states, become accessible. For argon, 17 bound states and 36 continuum states are included. This absorption potential also includes inner shell ionization. In all cases, the experimental energy threshold has been used rather than the calculated one. This state-by-state construction of the potential will, in principle, allow one to introduce an independent term to account for positronium formation but so far this has not been carried out. Differential elastic cross sections are directly given by the calculated complex scattering phase shifts. Some results for the differential cross sections for positron scattering from argon at incident energies ranging from 1 to $10000 \mathrm{eV}$ are shown in figure 3. Results for the total cross sections, excluding the positronium formation contribution, are shown in figure 2. As may be seen from this latter figure, above the positronium region, the agreement with experiment is excellent.

\section{Input data set}

After a detailed analysis of the available cross-section data and the results of the experiments and calculations mentioned in the previous sections, a complete set of recommended positronAr cross-section data has been derived. These recommended data are shown in table 1 . Where possible, experimental data have been considered preferentially, i.e. for low-energy (below $60 \mathrm{eV}$ ) total scattering and integral elastic cross sections as well as positronium formation and direct ionization cross sections. Integral elastic cross sections above $60 \mathrm{eV}$ and differential cross sections for the whole energy range have been derived from our calculations, using the $a b$ initio model potential results for the lower energies and the optical potential approach to extrapolate data up to $10 \mathrm{keV}$. By considering the experimental errors and the general level of agreement between the available data, a general uncertainty of $10 \%-15 \%$ can be estimated for our recommended data in table 1. By multiplying the recommended total scattering cross sections by the ratio of the inelastic to total scattering cross sections derived from the calculations, the corresponding integral inelastic cross sections have been derived. By subtracting the total ionization cross 

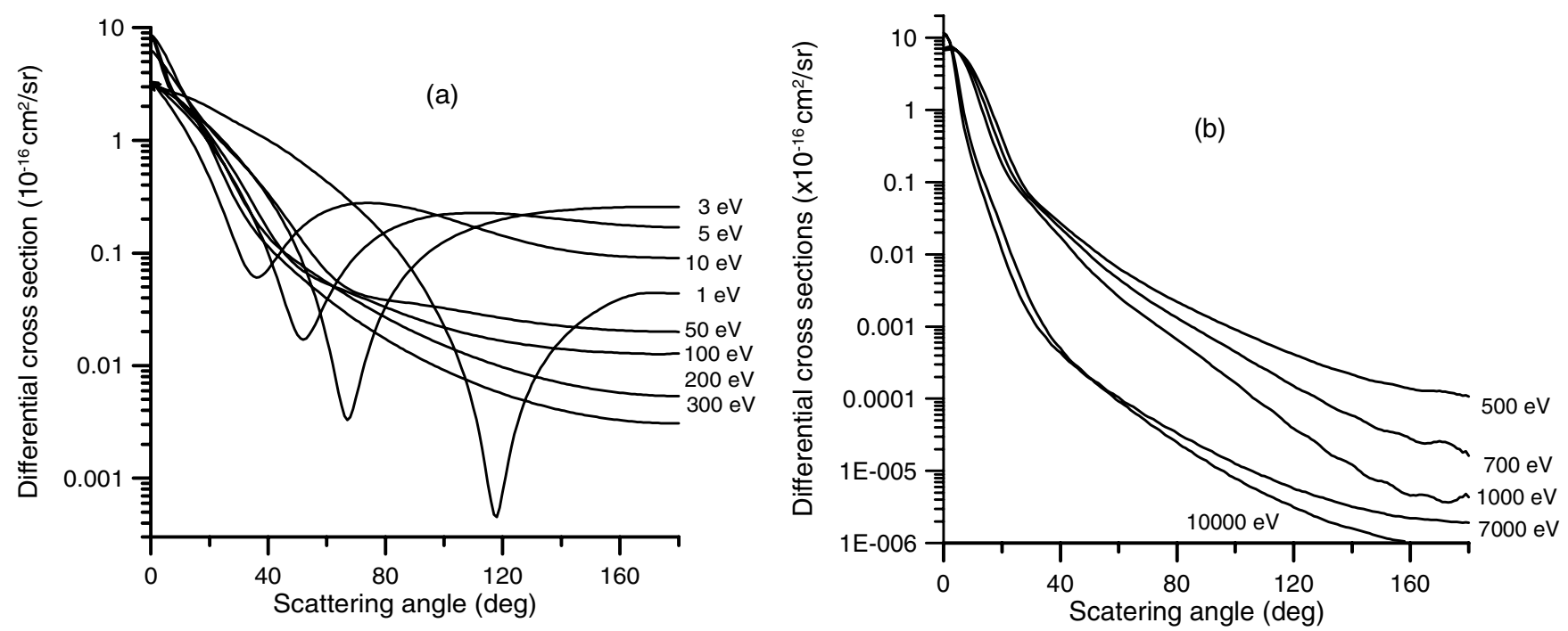

Figure 3. Representative differential cross sections for elastic positron scattering from Ar. (a) Low-energy calculation with the ab initio absorption potential (see section 4.2) and (b) high-energy calculation with the optical potential based on a quasi-free electron model (see section 4.1).

Table 1. Set of recommended input interaction cross sections (in $10^{-20} \mathrm{~cm}^{2}$ ) for positrons in Ar.

\begin{tabular}{|c|c|c|c|c|c|}
\hline Energy $(\mathrm{eV})$ & TCS & Elastic & Positronium & Ionization & Excitation \\
\hline 0.1 & 19.8 & 19.8 & & & \\
\hline 0.15 & 16.3 & 16.3 & & & \\
\hline 0.2 & 14.2 & 14.2 & & & \\
\hline 0.3 & 11.6 & 11.6 & & & \\
\hline 0.4 & 9.92 & 9.92 & & & \\
\hline 0.5 & 8.66 & 8.66 & & & \\
\hline 0.7 & 6.85 & 6.85 & & & \\
\hline 1 & 5.10 & 5.10 & & & \\
\hline 1.5 & 4.25 & 4.25 & & & \\
\hline 2 & 4.08 & 4.08 & & & \\
\hline 3 & 3.96 & 3.96 & & & \\
\hline 4 & 3.89 & 3.89 & & & \\
\hline 5 & 3.75 & 3.75 & & & \\
\hline 7 & 3.65 & 3.65 & & & \\
\hline 10 & 4.70 & 3.66 & 1.04 & & \\
\hline 15 & 6.20 & 3.35 & 2.65 & & 0.100 \\
\hline 20 & 6.58 & 2.81 & 2.65 & 0.200 & 0.919 \\
\hline 30 & 7.15 & 2.57 & 2.60 & 0.77 & 1.21 \\
\hline 40 & 7.30 & 2.53 & 2.20 & 1.38 & 1.19 \\
\hline 50 & 7.20 & 2.47 & 1.70 & 1.95 & 1.08 \\
\hline 70 & 6.89 & 2.36 & 0.910 & 2.76 & 0.857 \\
\hline 100 & 6.08 & 2.11 & 0.240 & 3.20 & 0.533 \\
\hline 150 & 5.17 & 1.84 & & 2.85 & 0.479 \\
\hline 200 & 4.56 & 1.67 & & 2.43 & 0.458 \\
\hline 300 & 3.77 & 1.45 & & 1.87 & 0.448 \\
\hline 400 & 3.26 & 1.30 & & 1.51 & 0.452 \\
\hline 500 & 2.89 & 1.18 & & 1.26 & 0.450 \\
\hline 700 & 2.39 & 1.00 & & 0.975 & 0.406 \\
\hline 1000 & 1.93 & 0.842 & & 0.735 & 0.353 \\
\hline 1500 & 1.49 & 0.673 & & 0.557 & 0.260 \\
\hline 2000 & 1.24 & 0.575 & & 0.447 & 0.217 \\
\hline 3000 & 0.944 & 0.453 & & 0.328 & 0.163 \\
\hline 4000 & 0.774 & 0.380 & & 0.263 & 0.131 \\
\hline 5000 & 0.660 & 0.330 & & 0.222 & 0.108 \\
\hline 7000 & 0.516 & 0.264 & & 0.172 & 0.081 \\
\hline 10000 & 0.392 & 0.204 & & 0.131 & 0.0570 \\
\hline
\end{tabular}

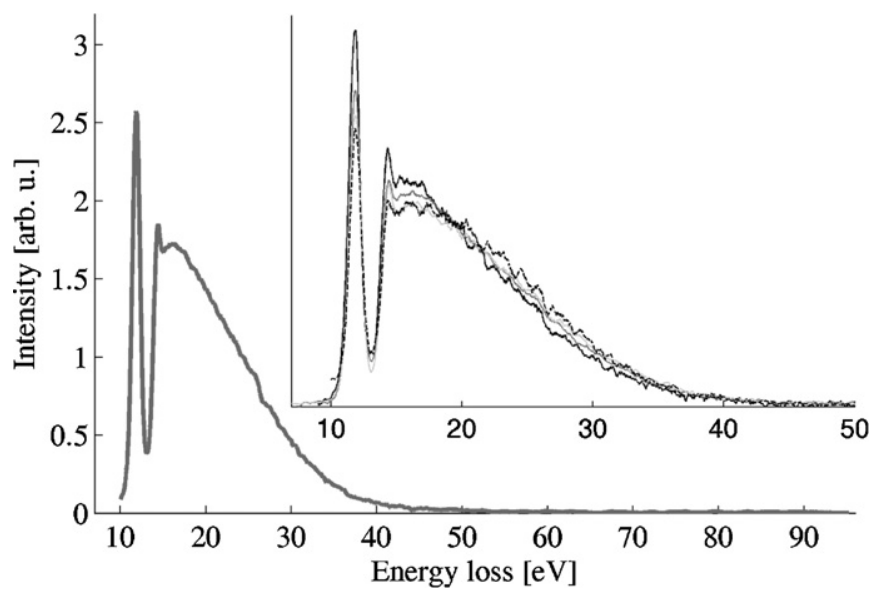

Figure 4. Average electron energy-loss spectrum obtained in the $\mathrm{keV}$ energy range in argon. Inset: average distribution measured at $1 \mathrm{keV}$ (black solid line), $1.5 \mathrm{keV}$ (grey solid line), $2 \mathrm{keV}$ (light grey solid line) and $3 \mathrm{keV}$ (dashed line).

section (direct ionisation plus positronium formation) from these integral inelastic data, total excitation cross sections have been obtained. Estimated errors for these last data are about $20 \%$.

Angular distribution functions for the elastic process have been derived directly from our calculations. Differential cross sections for elastic positron scattering from argon obtained with both theoretical methods, once normalized, defined the angle of the outgoing positrons after the collision. Representative examples of the present calculated differential cross sections from 1 to $10000 \mathrm{eV}$ are shown in figure 3. For inelastic scattering, we used the same angular distribution as for elastic scattering but allowed for the amount of energy transferred to the target to be set according to the procedure described in [24] for electrons.

As far as the energy-loss distribution function is concerned, figure 4 shows the detail of the experimental 


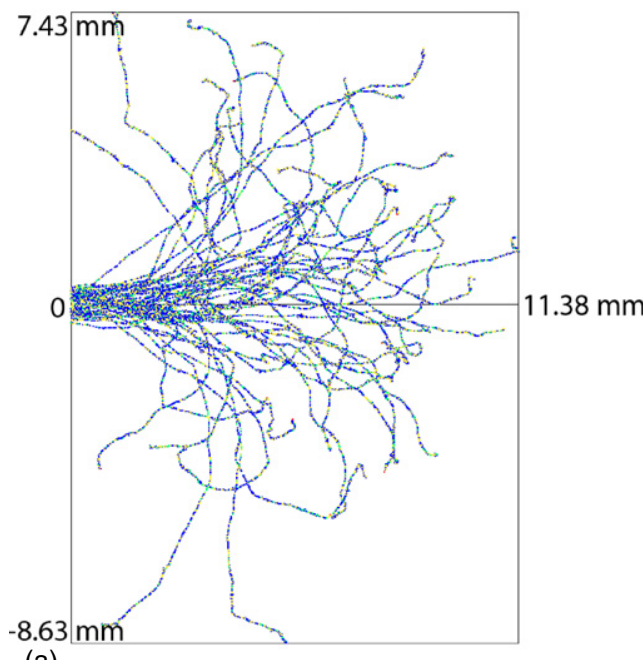

(a)

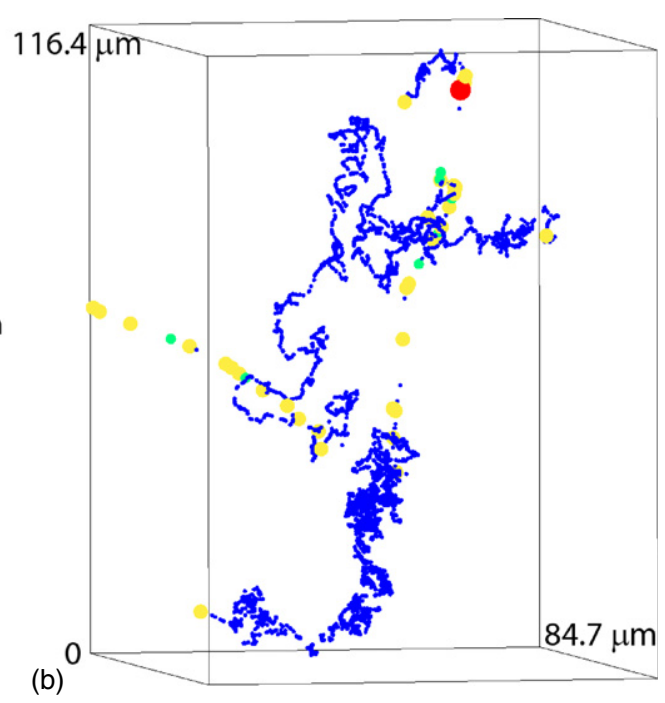

Figure 5. (a) 2D projection of 50 positron tracks in $\mathrm{Ar}$ (incident energy: $5 \mathrm{keV}$, pressure: $1 \mathrm{~atm}$, room temperature) simulated using LEPTS. (b) Expanded view of the final part of a single track. The positron annihilates shortly after forming positronium in the upper right corner. In both panels, the type of interaction undergone by the particle (positron or secondary electron) is colour coded as follows: blue (black), elastic scattering; cyan (dark grey), electronic excitation; yellow (medium grey), ionization; red (light grey), positronium formation.

energy-loss spectra observed for electrons scattered from argon at incident energies ranging from 1000 to $3000 \mathrm{eV}$. Each spectrum represents an average for scattering angles between $0^{\circ}$ and $10^{\circ}$. For energies above $100 \mathrm{eV}$, the energy-loss distribution is almost independent of the incident energy. This allows one to obtain a reasonable average energy distribution by following the averaging procedure, as a function of energy and scattering angle, described in [24]. By assuming that the main difference between positron and electron energy deposition mechanisms is the positronium formation process, and by considering that for these processes the positron tracks are terminated, we will use as an energy-loss distribution function for positrons that derived from the electron energyloss experiments.

\section{Single track simulations}

Single particle tracks have been simulated with a Monte Carlo code which has been described elsewhere $[31,32]$. It is an event-by-event simulation procedure, programmed in $\mathrm{C}++$, which is compatible with other general purpose Monte Carlo codes like GEANT4 [33]. Other related tools, such as the GEANT4-based architecture for medicine-oriented simulations (available from http://fismed.ciemat.es/GAMOS/), have been used to define the target geometries. Photon and high-energy electron (above $10 \mathrm{keV}$ ) tracks are then simulated with that general code, whereas low-energy electrons (below $10 \mathrm{keV}$ ) and positrons are treated by our low-energy particle track simulation (LEPTS) procedure.

For an incoming low-energy electron or positron, the free path in the medium is first sampled according to the total cross section corresponding to its collision energy. Once the location of an event is defined, partial cross sections determine whether an elastic or inelastic process is likely to take place and the appropriate interaction routine is called. For elastic collisions, since no energy is deposited in the medium, the programme samples the outgoing particle's angle according to the distribution established by the corresponding differential cross section. In the case of inelastic collisions, different sub-processes (with their relative frequency given by the corresponding partial cross section values) handle the different types of interactions that are accessible depending on the positron or electron energy, such as, ionization, excitation and positronium formation. First, the energy lost in the collision is determined from the energy-loss distributions (see figure 4). Subsequently, the particle's outgoing direction is sampled using the differential cross section expressed as a function of the momentum transfer (rather than the angle) as described in [34]. If ionization has taken place, a secondary electron is automatically generated and enters the simulation process with an energy given by the energy lost by the primary particle minus the ionization energy and moves in the direction obtained by applying linear momentum conservation. Positron and secondary electron tracks are then fully simulated with the same procedure. Finally, the interaction event has terminated and the electron or positron is ready to enter the next collision.

As an example of the simulation's output, figure 5 shows 50 positron tracks in $1 \mathrm{~atm}$ of $\mathrm{Ar}$ at room temperature. The positrons are released into the gas region through an aperture of $1 \mathrm{~mm}$ in diameter, with an initial energy of $5 \mathrm{keV}$, and are then tracked until thermalization (figure 5(a)). In figure 5(b), the final portion of a single track ending in positronium formation can be seen. Note the presence of secondary electrons which are visible as additional tracks, characterized typically by a large density of elastic scattering events, emerging from the main track. Due to the molecular-level description of all the interaction events achieved by the LEPTS model, a wide range of detailed information becomes available apart from the mere track structure. By making use of these additional data, table 2 shows useful results corresponding to figures 5(a) and (b). It displays the total energy deposited in both cases (entire 
Table 2. Dosimetric data obtained from the simulation of 50 positron tracks in $\operatorname{Ar}$ (1 atm pressure at room temperature) with an initial energy of $5 \mathrm{keV}$ (see text). The number and type of interaction events registered are given for the whole area (50 tracks) and for a zoom region as shown in figure 5 . The last two rows contain information about the corresponding energy deposition.

\begin{tabular}{lll}
\hline & 50 tracks & $\begin{array}{l}\text { End of single } \\
\text { track }\end{array}$ \\
\hline Volume & $2767.6 \mathrm{~mm}^{3}$ & $0.00103 \mathrm{~mm}^{3}$ \\
Total number of interactions & 1427197 & 5191 \\
Number of elastic collisions & 1396555 & 5142 \\
Number of ionizations & 21468 & 39 \\
Number of excitations & 9131 & 9 \\
Number of positronium & 43 & 1 \\
$\quad$ formations & $246286 \mathrm{eV}$ & $367 \mathrm{eV}^{-1}$ \\
Energy imparted & $0.0178 \mathrm{~J} \mathrm{~kg}$ & $71.6 \mathrm{~J} \mathrm{~kg}^{-1}$ \\
\hline Specific energy (energy/mass) & &
\end{tabular}

simulated area and zoom region) along with the frequencies of the different interactions that have taken place. It is difficult to determine the total error associated to the output of the simulation. Concerning the statistical errors of the Monte Carlo procedure, by increasing the number of events, they could be reduced even below $1 \%$, but we should note that uncertainties in the input data (see section 5) are directly transmitted to the results of the simulation. The availability of both types of information-energy deposition as well as the type of event produced at a given interaction point-is crucial to characterize induced damage in nano-sized volumes, as it is demanded in nanodosimetry.

\section{Conclusions}

We have presented two alternative theoretical approaches for obtaining cross sections for positron scattering from argon. While the first model uses a 'quasi-free' absorption potential, the second one describes inelastic scattering via an $a b$ initio absorption potential. After an analysis of the available experimental data and their level of agreement with the present calculations, a set of interaction cross sections (total scattering cross section, elastic scattering, positronium formation, direct ionization and excitation) for positrons in argon was recommended. The accuracy estimated for this recommended data in table 1 is typically $10 \%-15 \%$. The recommended data set can now be used together with theoretical differential cross sections and an energy-loss distribution, based on electron experimental data, in order to simulate positron trajectories in Ar with the LEPTS code. The detailed output provided by the LEPTS programme, including not only energy deposition data but also information about the type of event produced in each collision, facilitates the nanodosimetric investigation of the effects induced by positron irradiation of matter.

In order to expand the application range of the present simulation procedure, we will continue to study other materials focussing especially on targets of biomedical interest.

\section{Acknowledgments}

This work has been carried out with partial financial support of the Spanish Ministerio de Ciencia e Innovación (Project FIS2009-10245), the European Science Foundation (COST Actions CM0601 and MP1002) and the Australian Research Council's Centre of Excellence Program. The authors acknowledge the important contribution of Professor Gaetana Laricchia by her summarizing the positron interaction cross sections measured at University College London.

\section{References}

[1] Catana C, Wu Y, Judenhofer M S, Qi J, Pichler B J and Cherry S R 2006 J. Nucl. Med. 471968

[2] Sánchez-Crespo A, Andreo P and Larsson S A 2004 Eur. J. Nucl. Med. Mol. Imaging 3144

[3] Perkins S T, Cullen D E and Seltzer S M 1991 LLNL Evaluated Electron Data Library 31 UCRL-50400

[4] Blanco F and García G 2002 Phys. Lett. A 295178

[5] Jones A C L, Makochekanwa C, Caradonna P, Slaughter D S, Machacek J R, McEachran R P, Sullivan J P, Buckman S J, Stauffer A D, Bray I and Fursa D V 2011 Phys. Rev. A 83032701

[6] Sullivan J P, Makochekanwa C, Jones A, Caradonna P, Slaughter D S, Machacek J, McEachran R P, Mueller D W and Buckman S J 2011 J. Phys. B: At. Mol. Opt. Phys. 44035201

[7] Sullivan J P, Marler J P, Gilbert S J, Buckman S J and Surko C M 2001 Phys. Rev. Lett. 87073201

[8] Laricchia G 2010 private communication

[9] Zecca A, Chiari L, Trainotti E, Fursa D, Bray I, Sarkar A, Chattopadhyay S, Ratnavelu K and Brunger M J 2012 J. Phys. B: At. Mol. Opt. Phys. 45015203

[10] Suvakov M, Petrovic Z Lj, Marler J P, Buckman S J, Robson R E and Malovic G 2008 New J. Phys. 10053034

[11] White R D, Dujko S, Robson R E, Petrovic Z Lj and McEachran R P 2010 Plasma Sources Sci. Technol. 19034001

[12] Muñoz A et al 2008 J. Phys. Conf. Ser. 133012002

[13] Sanz A G, Fuss M C, Muñoz A, Blanco F, Limão-Vieira P, Brunger M J, Buckman S J and García G 2012 Int. J. Rad. Biol. 8871

[14] García G, Roteta M, Manero F, Blanco F and Williart A 1999 J. Phys. B: At. Mol. Opt. Phys. 321783

[15] García G and Manero F 1998 Phys. Rev. A 571069

[16] Sullivan J P, Jones A, Caradonna P, Makochekanwa C and Buckman S J 2008 Rev. Sci. Instrum. 79113105

[17] Charlton M 1985 Rep. Prog. Phys. 48737

[18] Moxom J, Laricchia G and Charlton M 1995 J. Phys. B: At. Mol. Opt. Phys. 281331

[19] Jacobsen F M, Frandsen N P, Knudsen H, Mikkelsen U and Schrader D M 1995 J. Phys. B: At. Mol. Opt. Phys. 284691

[20] Bluhme H, Knudsen H, Merrison J P and Nielsen K A 1999 J. Phys. B: At. Mol. Opt. Phys. 325835

[21] Laricchia G, Armitage S, Leslie D E, Szhuinska M and Van Reeth P 2003 Rad. Phys. Chem. 6821

[22] Marler J P, Sullivan J P and Surko C M 2005 Phys. Rev. A 71022701

[23] Campeanu R I 2009 Nucl. Instrum. Methods B 267239

[24] Fuss M C, Muñoz A, Oller J C, Blanco F, Hubin-Franskin M-J, Almeida D, Limão-Vieira P and García G 2010 Chem. Phys. Lett. 486110

[25] Blanco F and García G 2003 Phys. Rev. A 67022701

[26] Reid D D and Wadehra J M 1994 Phys. Rev. A 504859

[27] Reid D D and Wadehra J M 1996 J. Phys. B: At. Mol. Opt. Phys. 29 L127 
Reid D D and Wadehra J M 1997 J. Phys. B: At. Mol. Opt. Phys. 302318

[28] Charlton M and Humberston J W 2001 Positron Physics (Cambridge: Cambridge University Press)

[29] Chen S, McEachran R P and Stauffer A D 2008 J Phys B: At. Mol. Opt. Phys. 41025201

[30] McEachran R P, Ryman A G and Stauffer A D 1979 J. Phys. B: At. Mol. Opt. Phys. 121031

[31] Roldán A M, Pérez J M, Blanco F, Williart A and García G 2004 J. Appl. Phys. 95 5865-70
[32] Muñoz A, Pérez J M, García G and Blanco F 2005 Nucl. Instrum. Methods A 536 176-89

[33] Agostinelli S et al (for the Geant4 Collaboration) $2003 \mathrm{Nucl}$. Instrum. Methods A 506 250-303

[34] Muñoz A, Blanco F, García G, Thorn P A, Brunger M J, Sullivan J P and Buckman S J 2008 Int. J. Mass Spectrom. 277 175-9

[35] García G, Roteta M, Manero F, Blanco F and Williart A 1999 J. Phys. B: At. Mol. Opt. Phys. 321783 